\title{
Modeling and Simulation Capacity Analysis of MIMO Wireless Sensor Network
}

\author{
Rajkumar A. Veer ${ }^{1}$, Dr L C Siddanna Gowd ${ }^{2}$ \\ Research Scholar, Faculty of ECE Dept, Bharath University, Chennai, T.N ${ }^{1}$ \\ Professor, Faculty of ECE Dept, AMSEC, Namakkal, T.N²
}

\begin{abstract}
In conventional wireless communication system the problem of estimating the parameters such as coherence time, coherence band width etc. is still not accurate. This problem is mainly due to the fact that the mobile receiver can be in motion and also received signals can arrive along multiple reflected paths. In this work, the channel modeling, optimal location reflectors and scatterers with respect to the location of transmitter and receiver and the optimal valve of angular range with the direction of motion of the mobile receiver are to be explored. The effect of the multipath fading both over time and frequency is also to be considered. The algorithms and schemes proposed in this paper shall benefit in the (i) optimal location of scatterers around a receiver moving at a variable speed (ii) realistic modeling of MIMO fading channels overcoming the conventional assumption that fading coefficients between different transmit and receive antennas are independent random variables.
\end{abstract}

Keywords: Multipath fading, Mobility models, MIMO fading channel, Channel uncertainty.

\section{INTRODUCTION}

Typically, the channel tap gains vary in time and the different dynamics operate at different time-scales. The usual assumption that communication takes place on a bandwidth ' $\mathrm{W}$ ' around a carrier frequency fc $(\mathrm{fc}>>\mathrm{W})$ need not hold good for Ultra-Wideband (UWB) communication systems [2] [22]. The typical transmitter band width is from $3.1 \mathrm{GHz}$ to $10.6 \mathrm{GHz}$ for UWB (as regulated by FCC)[18]. In this paper, it is proposed to determine the fastest time-scale rate of variation of the channel tap gains. The schemes proposed in this paper shall assist to (i) investigate different diversity techniques (operating both over time and frequency) those improve the performance. This is highly essential since there is a significant probability that a channel is in deep fade[3] (ii) study space diversity (i.e. both transmit and receive diversity schemes) and frequency diversity (i.e. signal carrier with ISI equalization and multiplexing schemes using orthogonal techniques) (iii) understand the impact of channel uncertainty the performance of diversity combing schemes (iv) perform signal detection in fading channels in an optimal manner (both non-coherent and coherent detection) (v) improve spectral efficiency by exploiting the degrees of freedom in orthogonal modulation schemes[15] (vi) devise improved coding and interleaving techniques and achieve diversity over time. This ensures that different parts of the code words experience independent fades. (vii) propose macro-diversity schemes for improved performance of wireless systems.

\section{METHODLOGY}

In this paper, it is proposed to formulate a universal criterion that works for all channel statistics and remains an optimal time-diversity scheme. The design of such a criterion requires the generalization of the symbol coding as they pass through correlated fades of the channel[6]. Whenever there is a strict delay constraint or coherence time is large then interleaving and coding over several coherence time periods is difficult. Hence, in this research, Antenna diversity schemes are proposed and multiple antennas are to be placed at the transmitter and receiver. Therefore, it is proposed in this work to create independent signal paths by optimally placing the antennas (space time coding) [12].

In this paper, the maximum likelihood (MLSD) Rake receiver detection of a sequence based on a received vector is proposed. This detection is to be done in the optimal sense such that the solution is recursive in ' 0 ' and the problem need not be solved from beginning for every symbol time. Since the performance of a coherent rake receiver is better compared to MLSD[17], this scheme is preferred in this work. However, to assist the design of such a receiver for practical use, the channel taps are pre estimated and tracked in this work. A novel hybrid technique that integrates the pilot-based and decisiondirected scheme for channel estimation is proposed in this work. This is particularly important in scenarios where there are many diversity branches but only a small fraction of signal energy is received along each branch.

\section{BENEFITS OF THE WORK}

The following are the benefits of the proposed work:

(i) More sophisticated schemes with higher diversity gain can be designed. 
IARJSET

(ii) Better/Proper focus of transmit energy on smaller
number of diversity branches and thereby an effective increase in SNR is possible.

(iii) The work can minimize the gap between coherent and non coherent schemes and perform effective combination of signals along each branch.

(iv) The work assists in determining the optimal state information that helps in finding high SNR performance for chosen number of independent branches of diversity.

\section{PREVIOUS WORK}

[1] deals with Multiple Input Multiple Output (MIMO) systems in current and future wireless telecommunications systems. The advantages of adaptive antenna arrays, diversity techniques and water filling techniques are described. The Possibilities of MIMO systems for the increase of channel capacity, reliability and spectrum efficiency is also presented. In [5] the joint channel estimation and resource allocation problem in MIMO systems with noisy channel estimation at the receiver side and partial CSI, in the form of covariance feedback, available at the transmitter side is presented. Correlated MIMO channels with block fading, where each block is divided into training and data transmission phases is considered. In the first phase of this work the single-user scenario is analyzed and the channel estimation and data transmission parameters that jointly optimize the achievable data rate of the system are obtained. The results are then extended to the multiple access channel. It is showed that the optimum training signals of the users should be orthogonal in time. For the data transmission phase, an iterative algorithm that updates the parameters of the users in a round-robin fashion is proposed. In particular, the algorithm updates the training and data transmission parameters of a user, when those of the rest of the users are fixed, in a way to maximize the achievable sum-rate in a multiple access channel; and iterates over users in a round-robin fashion.

In [10] it is shown that the Co-Channel Interference (CCI) caused by different Multiple Input Multiple Output (MIMO) signaling schemes impact the same or other MIMO schemes in different ways. Due to the symbol-bysymbol processing in a desired Single Input Multiple Output (SIMO) link, it is shown in their work that an interfering Space-Time Block Code (STBC) link may become equivalent to an interfering Spatial Multiplexing (SM) link. Using this knowledge and understanding, it is possible to propose an interference cancellation receiver robust to different types of MIMO interferers at cell edge for the Downlink (DL) of cellular systems[13]. The receiver systematically performs a multiple symbol processing: this is the appropriate processing when the signal of interest or the signal of interferer is correlated across symbols, which is the case for STBC transmission. Their work has also evaluated different link combinations in terms of Signal to Interference and Noise Ratio (SINR) statistics and Bit Error Rate (BER) performance in cellular
Orthogonal Frequency Division Multiple Access (OFDMA) systems. It is established in their work that the proposed multiple-symbol linear interference cancellation receiver performs satisfactorily when any kind of single 'logical' stream MIMO scheme is present as interferer. This is shown in [19][11]. In this reference, the tradeoffs between diversity, interference cancellation and spatial multiplexing in MIMO systems are discussed, and a comparison among optimum combining (OC), maximumratio combining (MRC) and interference cancellation for different numbers of receive antennas are presented. Then, the two mandatory MIMO profiles in the IEEE specifications (Alamouti's STC and the $2 \times 2$ spatial multiplexing scheme) are discussed and comparison between them is presented[20]. The analysis obtained using the ITU pedestrian B channel in ideal conditions indicates that the two schemes lead to similar performance when they are operated at the same spectral efficiency[14]. MIMO systems may achieve spatial multi-user access using singular value decomposition (SVD) MIMO techniques. The MIMO OFDM systems multiplex the users both in the frequency and the spatial domains.

Hence, the co-channel interference caused by the subcarrier reuse may lower the system's performance[8]. For MIMO OFDM systems with co-channel interference, the combination of power control with adaptive modulation is desirable to reduce the effect. This is proposed in [25] where an allocation algorithm is proposed by starting an OFDMA-based allocation procedure. Thus, the amount of the co-channel interference is mitigated by the sub-carrier allocation. The scheme used a neighborhood search scheme to obtain additional power efficiency. However, the proposed scheme has the limitation on the affordable maximum data rate even without transmit power constraint[7]. The work presents an enhanced version of the scheme to remedy the problem. The proposed scheme aims to minimize the total user transmit power while satisfying the required data rate, the maximum transmit power constraint, and the bit error rate of each user.

\section{EXPERIMENTATION}

This section describes the simulation setup used for the implementation and the experimentation part used to investigate different diversity schemes. The protocols have been implemented under the mobility patterns such as (i) study of optimal location of scatterers around a receiver (Random waypoint), Realistic modeling of MIMO fading channels (Random Walk) and channel uncertainty effect model (Random Direction).

\section{A. Simulation Setup}

In order to study and analyze the performance of the different mobility models with respect to the various parameter constraints, it is necessary to simulate a time diversity network[9]. The traffic patterns used in this simulation is with 50 nodes and two categories of connections such as 10 and 20. The number of packets 
IARJSET

chosen for transmission is 1 or 4 packets for each category The Random Waypoint Mobility Model includes pauses of connections. The lists of parameters that are considered between changes in direction and/or speed. A Mobile node for the various models in the simulation is listed in Table begins by staying in one location for a certain period of 1. From the simulation each mobility model is grouped on the basis of Speed $(10 \mathrm{~m} / \mathrm{s}$ and $20 \mathrm{~m} / \mathrm{s})$, Number of packets (1 pkt/sec and $4 \mathrm{pkt} / \mathrm{sec})$ and Simulation Area $(400 \mathrm{~m} \mathrm{x}$ $400 \mathrm{~m}$ and $1000 \mathrm{~m} \times 600 \mathrm{~m})$.

Table 1 Parameters for Random Mobility Model

\begin{tabular}{|l|l|l|l|}
\hline $\begin{array}{l}\text { Mobility } \\
\text { Model }\end{array}$ & $\begin{array}{l}\text { Speed } \\
(\mathrm{m} / \mathrm{s})\end{array}$ & $\begin{array}{l}\text { Pause Time } \\
(\mathrm{Sec})\end{array}$ & $\begin{array}{l}\text { Travel Time } \\
(\mathrm{Sec})\end{array}$ \\
\hline $\begin{array}{l}\text { Random } \\
\text { Waypoint }\end{array}$ & 10,20 & $\begin{array}{l}100, \quad 200, \\
500,900\end{array}$ & - \\
\hline $\begin{array}{l}\text { Random } \\
\text { walk }\end{array}$ & 10,20 & - & 20,40 \\
\hline $\begin{array}{l}\text { Random } \\
\text { Direction }\end{array}$ & 10,20 & $\begin{array}{l}100, \quad 200, \\
500,900\end{array}$ & - \\
\hline
\end{tabular}

In this work, the Random Waypoint, Random Walk and Random Direction mobility models has been implemented. The performance of the wireless system has been analyzed with respect to speed, node density and traffic load. Based on the analysis the packet delivery ratio and end-to-end delay is also calculated. The Simulation results obtained in the experimentation is discussed and analyzed in section 6 .

\section{IMPLEMENTATION OF THE MPBILITY MODELS}

The performance of the protocols in this work is done using the mobility models such as Random Waypoint, Random Walk and Random Direction. These models with various parameters reflect the realistic traveling pattern [4] of the mobile nodes. The following section explains all the three models with the traveling pattern of the mobile nodes and how the nodes are distributed during the simulation time.

\section{A. Optimal location of scatterers around a receiver (Random Waypoint)}

This model [16] is a commonly used mobility model in the simulation of wireless networks. It is assumed in this model that the spatial distribution of network nodes move according a nonuniform distribution [3].

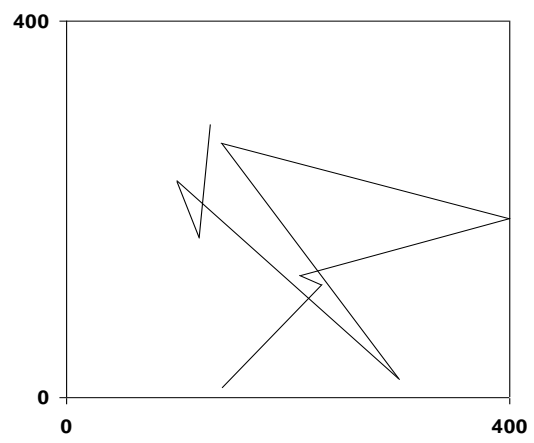

time (i.e. pause). Once this time expires, the mobile node chooses a random destination in the simulation area and a speed that is uniformly distributed between [0, MAXSPEED]. The mobile node then travels toward the newly chosen destination at the selected speed. Upon arrival, the $\mathrm{MN}$ takes another break before starting the process again.

The traveling pattern of a mobile node using the Random Waypoint model is shown in figure 2. The $\mathrm{x}$ and $\mathrm{y}$ axis represent distance in meters. This pattern is generated for 1000 seconds of simulation with maximum pause time 100 seconds, and with maximum speed of $20 \mathrm{~m} / \mathrm{s}$. The simulation wireless network area considered is $400 \mathrm{x} 400$ meters. From the mobility pattern of Figure 1, it may be seen that most of the time the mobile nodes try to find some location near the centre of the simulation area. Thus the average number of neighbors for the mobile nodes in the centre of the area will be higher than that of the simulation area.

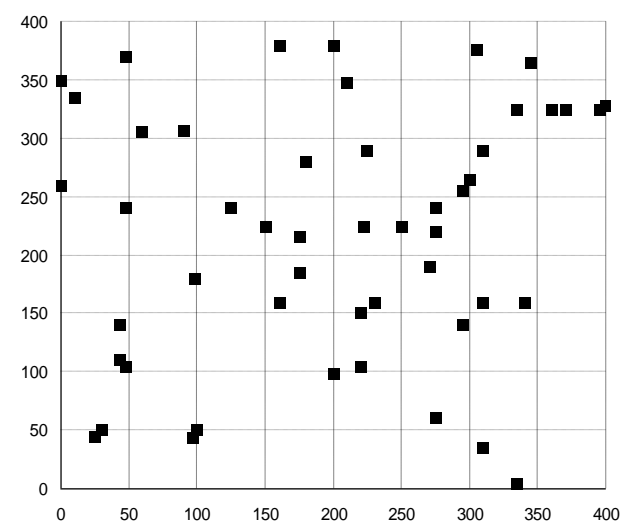

Figure 2 Random Waypoint Initial Distribution of Nodes

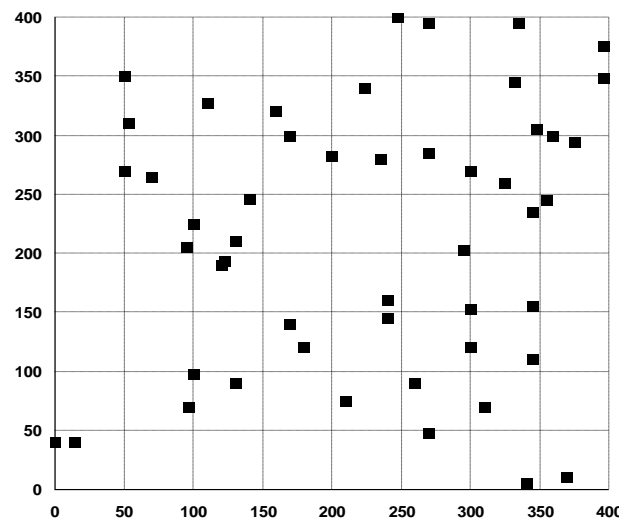

Figure 3 Random Waypoint Node Distribution after 500 Seconds

Figure 2 shows the initial positions of the 50 mobile nodes, whose locations are chosen randomly within the simulation area. Figure 3 shows positions of the nodes after 500 seconds of the simulation. It can be seen that the 
IARJSET

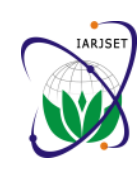

International Advanced Research Journal in Science, Engineering and Technology

National Conference on Innovative Applications and Research in Computer Science and Engineering (NCIARCSE-2017) AGTI's Dr. Daulatrao Aher College Engineering, Vidyanagar Extension, Karad

Vol. 4, Special Issue 4, January 2017

density of the nodes in the simulation area does not change heavily even after a long time.

\section{B. Realistic modeling of MIMO fading channels (Random Walk Model)}

This Mobility Model [23] [24] has proven to be one of the most widely used mobility models. In this mobility model, a mobile node moves from its current location to a new location by randomly choosing both direction and speed. The conventional assumption that fading coefficients between different transmit and receive antennas are independent random variables [21] is removed in this model. The new speed and direction are both chosen from pre-defined ranges, [min-speed, max-speed] and [0, 2*pi] respectively. Each movement in the MIMO fading channel Mobility Model occurs in a constant time interval ' $t$ ' at the end of which a new direction and speed are calculated. If the $\mathrm{MN}$ reaches the boundary before the completion of the travel time, it will evaluate the bouncing angle from the direction in which it was traveling. Then it will travel in the bouncing angle direction with the already chosen speed for the remaining time to travel. At the end of the travel time, the MN chooses a new direction and speed and continues to travel. The MNs do not stop anywhere, i.e. this model does not include pause time for the mobile nodes.

The figure 5 shows the mobility pattern of a mobile node based on the random walk mobility model. The node travels with a speed $10 \mathrm{~m} / \mathrm{s}$ and travels for a maximum of 20 seconds with the randomly chosen speed and direction.

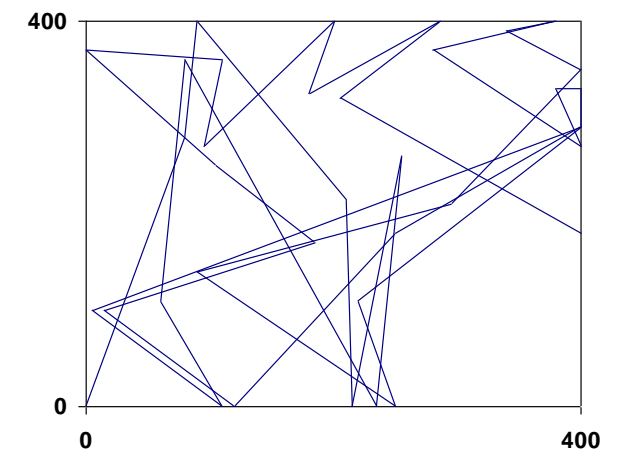

Figure 4 Node Movement in Random Walk

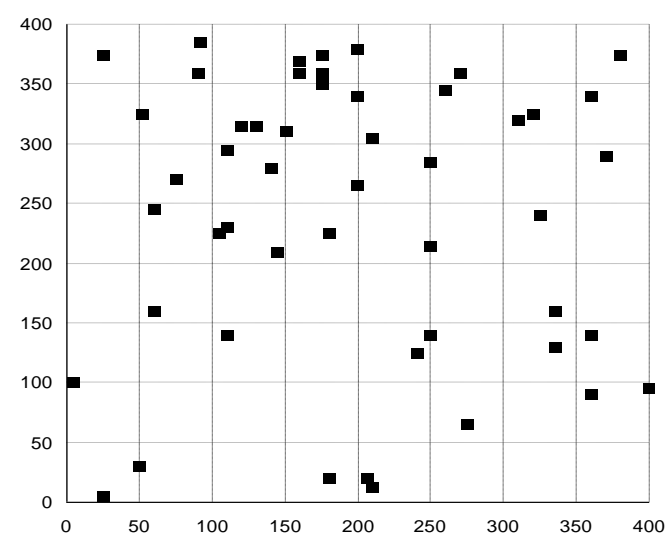

Figure 5 Random Walk Initial Distribution of Nodes

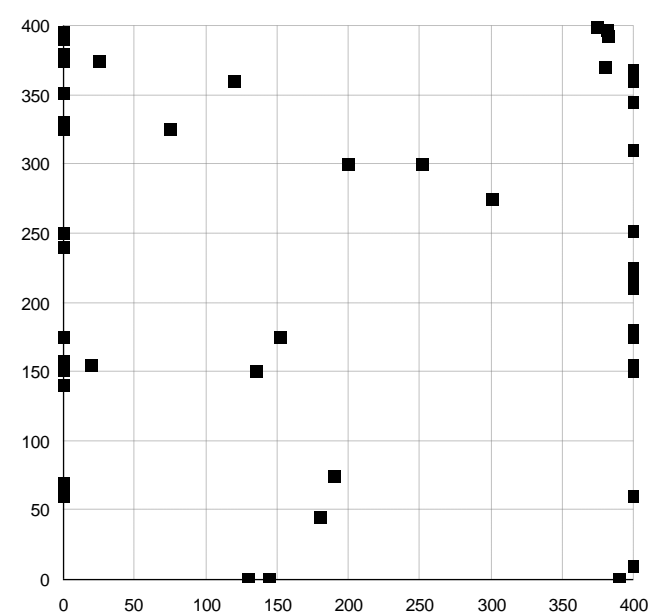

Figure 6 Random Walk Node Distribution after 500

Seconds

The distribution of nodes in the simulation at the initial stage is uniform as in Figure 5. When the travel time is set to a large value, (for e.g., 20 seconds), the nodes are allowed to travel to long distance with a high velocity. Thus the distribution of nodes near the centre of the area becomes sparse and near the boundaries it will be dense as shown in the Figure 6. This will increase the number of hop distance between the source destination pairs and thus increase the end-to-end delay in delivering the packets. When the travel time is kept as small value then the movement pattern is a random roaming pattern restricted to a small portion of the simulation area thus forming a semi-static network.

\section{Channel uncertainty effect model (Random Direction)}

In the channel uncertainty effect (study) model, the MNs choose a random direction in which to travel, instead of a random destination. After choosing a random direction, a $\mathrm{MN}$ travels to the border of the simulation area in that direction with a randomly chosen speed in the range $\left[\right.$ Speed $_{\min }$, Speed $\left._{\max }\right]$. As soon as the boundary is reached the MN stops for a certain period of time (pause time), chooses another angular direction, speed and continues the process. The MNs are allowed to stop only at the boundaries.

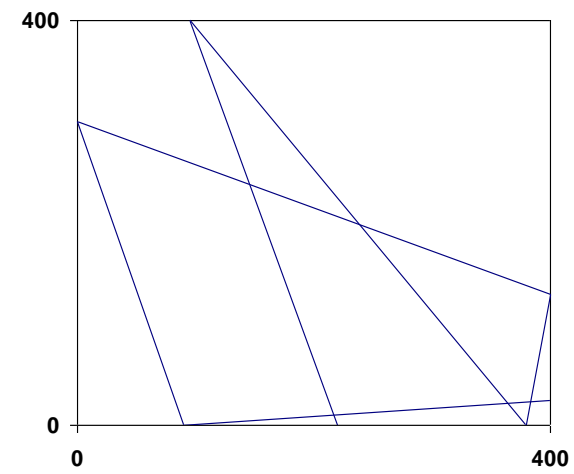

Figure 7 Node Movement in Random Direction 
IARJSET

The Figure 7 shows the mobility pattern of a mobile node (ii) based on the random direction mobility model with in an (iii) area of $400 \times 400$ meters moving with a maximum speed (iv) of $20 \mathrm{~m} / \mathrm{s}$ and using maximum pause time of 100 seconds. The mobile nodes travel to boundaries thus not forming any density wave anywhere in the network. Due to the pause times assigned to nodes that are reaching the boundaries, most of the time, most of the nodes are in the boundary or very close to the boundary. Thus the number of hop distance between the source-destination pairs will increase and the end-to-end delay also increases. This implies higher diversity gain.

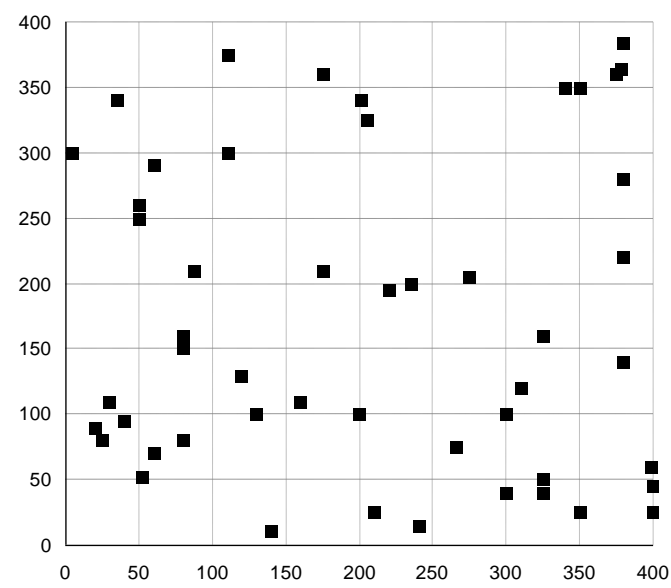

Figure 8 Random Walk Initial Distribution of Nodes

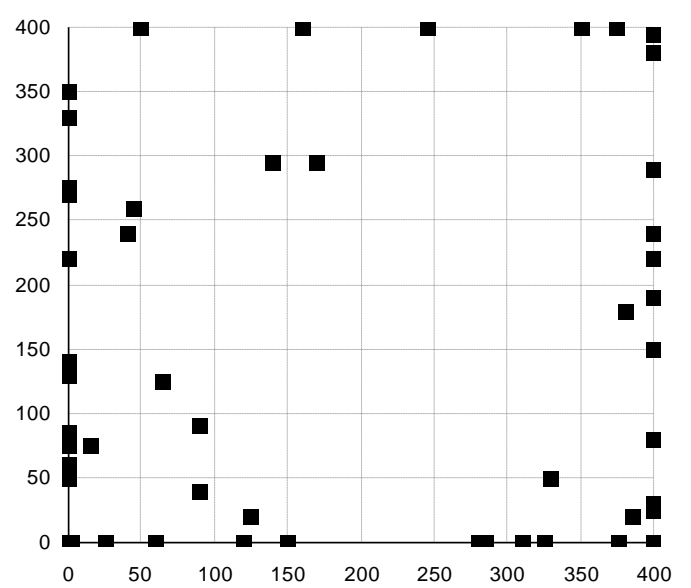

Figure 9 Random Walk Node Distribution after 500 Seconds

The sparse distribution of nodes can be seen from the distribution of nodes at the time 500 seconds as shown in Figure 8 against the initial distribution of nodes as in Figure 9.

\section{INFERENCE FROM SECTION 6.3}

The discussion in section 6 helps to achieve the following:

(i) The transmitter to optimize its power allocation or input covariance structure across antennas
Allocate equal power to each transmit antenna

Maximizes mutual information

Maximum data rate that can be transmitted over the channel is averaged over all channel realizations

(v) Angular resolvability is only intrinsically limited by the length of the array

(vi) Use of longer antenna array facilitate the transmitter to focus the energy along a desired direction and (vii) it can better spatially multiplex information to the multiple receive antennas

(vii) Achieve additional degrees of freedom without putting either the transmit antennas or the receive antennas far apart

(viii) The benefit of multipath is fully exploited such that degrees of additional freedom can be achieved.

\section{CONCLUSION}

This paper presents models to investigate different diversity schemes and provides a study of merits and demerits of different schemes. The work is highly relevant since there is a greater probability that a channel can be in deep fade. Also, channel uncertainty effects have been studied and schemes to suggest minimization of ISI are proposed. The techniques presented in this paper can assist in optimal signal detection both coherent and noncoherent.

\section{REFERENCES}

[1] Zentner. R, Nagy R, Zentner E: „Elliptical Single- bounce Model for MIMO Channel Simulations", COST273, Germany, 2004.

[2] Gesbert, David; Akhtar, Jabran. Breaking the barriers of Shannon's capacity: An overview of MIMO wireless system. Telektronikk: 5364, 2008.

[3] G. J. Foschini, M. J. Gans, , On Limits of wireless Communications in a Fading Environment", Wireless Personal Communications, 6, pp. 311-335, 1998.

[4] W. Yu, W. Rhee, S. Boyd, and J. M. Cioffi. Iterative water-filling for Gaussian vector multiple access channels. IEEE Transactions on Information Theory, 50(1):145-151, January 2004.

[5] A. Soysal and S. Ulukus. Optimum power allocation for single-user MIMO and multiuser MIMO-MAC with partial CSI. IEEE Journal on Selected Areas in Communications, 25(7):1402-1412, September 2007.

[6] Cho, M., Cho, H., Ro, S., and Hong, D.: 'A novel time spreading method for down-link OFDM-coded division multiplexing systems'. Proc. IEEE VTC, April 2003, pp. 764-768.

[7] Sanzi, F.: 'Comparison of bit error rate and convergence of four different iterative receivers for wireless OFDM-CDM', Int. J. Electron. Commun., 2005, 59, (3), pp. 166-176.

[8] M.I. Rahman, E. de Carvalho \& R. Prasad, "Impact of MIMO CoChannel Interference," in proc. 18th IEEE PIMRC'07, Athens, Greece, 3-7 September 2007.

[9] A.J. Paulraj, R. Nabar \& D. Gore, Introduction to Space-Time Wireless Communications, 1st ed. Cambridge University Press, September 2003.

[10] G. J. Foschini and M. J. Gans, "On limits of wireless communications in a fading environment when using multiple antennas," Wireless Personal Comm., vol. 6, pp. 311-335, March 1998.

[11] R. V. Nee and R. Prasad, OFDM for Wireless Mtultimedia Commucnications. Artech House, 2000.

[12] G. G. Raleigh and J. M. Cioff, "Spatio-temporal coding for wireless communications," IEEE Transactions on Commnnunications, vol. 46, pp. 357-366, March 1998. 
IARJSET

[13] Joseph M. Kahn and John R. Barry, "Wireless infrared communications," Proceedings of the IEEE, vol. 85, no. 2, pp. 265298, Feb. 1997.

[14] Y. Alqudah, M. Kavehrad, and S. Jivkova, "Optical wireless multispot diffusing; a mimo configuration," IEEE International Conference on Communications, vol. 6, pp. 3348 - 3352, June 2004.

[15] M. Simon and V. Vilnrotter, "Alamouti-type space-time coding for freespace optical communication with direct detection," IEEE Trans. On Wireless Communications, vol. 4, no. 1, pp. 35-39, 2005.

[16] IEEE 802.16-2005: IEEE Standard for Local and Metropolitan Area Networks - Part 16: Air Interface for Fixed and Mobile Broadband Wireless Access Systems - Amendment 2: Physical Layer and Medium Access Control Layers for Combined Fixed and Mobile Operation in Licensed Bands, February 2006.

[17] A. Goldsmith, Wireless Communications, Cambridge University Press, 2005

[18] S. Verd'u, "Spectral Efficiency in the Wideband Regime", IEEE Trans. Inform. Theory, vol. 48, no. 6, pp. 1319-1343, June 2002.

[19] V. Sethuraman, and B. Hajek, "Capacity per Unit Energy of Fading Channels with a Peak Constraint", in Proc. IEEE Int.Symp. on Inform. Theory, 2003.

[20] L. Zheng, M. M'edard, D. Tse, and C. Luo, "On Channel Coherence in the Low SNR Regime", in Proc. Allerton Conf., 2003.

[21] I.E. Telatar, "Capacity of multi-antenna gaussian channels",European transactions on telecommunications, vol. 10, pp. 585-595, Nov-Dec 1999.

[22] H. F. Chong, M. Motani and H. K. Garg, "A comparison of two achievable rate regions for the interference channel," Proceedings of USCD-ITA Workshop 2006, San Diego, CA, February 2006.

[23] Yang Han, See Ho Ting and Yong Liang Guan. A High Rate OpenLoop MIMO Multi-User Downlink Transmission System.

[24] A. Bury, J. Egle, and J. Lindner, "Diversity comparison of spreading transforms for multicarrier spread spectrum transmission," IEEE Trans.Commun., vol. 51, no. 5, pp. 774-781, May 2003.

[25] J. Z. Ying and K. B. Letaief, "An efficient resource-allocation scheme for spatial multi-user access in MIMO/OFDM systems," IEEE Trans. Comm., vol. 53, no.1, pp. 107- 116, Jan. 2005. 